\title{
Fracture de l'os hyoïde par hyperextension cervicale
}

\section{Hyoid Bone Fracture by Hyperextension of the Neck}

\section{Moulin · F. Heraud $\cdot$ E. Dubie}

Reçu le 21 octobre 2018; accepté le 12 janvier 2019

(C) SFMU et Lavoisier SAS 2019

\section{Introduction}

La fracture de l'os hyoïde est une fracture rare, en raison de sa protection par les éléments anatomiques alentours et de la mobilité de l'os [1-2]. Les mécanismes principaux en cause sont des traumatismes directs par strangulation ou lors d'accidents sur la voie publique. En raison de la faible incidence de ce type de fracture, il n'existe pas de recommandation sur le traitement [1-3]. Nous rapportons un cas de fracture de l'os hyoïde, diagnostiqué aux urgences dans les suites d'une chute à vélo sans impact direct sur la face antérieure du cou.

\section{Observation}

Une femme de 45 ans se présente aux urgences pour une chute à vélo, non casquée, à faible cinétique. Ses antécédents sont principalement marqués par une dissection vertébrale gauche et des migraines. Elle a pour seul traitement au long cours de l'aspirine $100 \mathrm{mg}$. La patiente déclare avoir chuté en avant par-dessus le guidon, avec réception sur le menton en hyperextension et sur les poignets. Le bilan radiologique retrouve une fracture de l'extrémité distale du radius droit avec avulsion de la styloïde radiale nécessitant une intervention chirurgicale. Au niveau facial, l'examen clinique retrouve des dermabrasions du menton et du philtrum. Aucune lésion visible au niveau de la gorge n'est à noter. Devant une symptomatologie pharyngée douloureuse intense et persistante (dysphagie, odynophagie avec sensation de striction) majorée à la palpation cervicale antérieure sans lésion visible à l'examen clinique, une nasofibroscopie est réalisée mais ne relève aucune anomalie. Il est alors réalisé un scanner cervical sans injection de produit de contraste qui met en évidence une fracture de l'os hyoïde (Fig. 1). Le reste de l'examen est sans particularité. Après avis ORL, le traitement choisi est une

\section{Moulin $(\bowtie) \cdot$ F. Heraud $\cdot$ E. Dubie}

Service des urgences, centre hospitalier Métropole Savoie, 505, faubourg Maché, F-73000 Chambéry, France e-mail : lauramoulin@hotmail.fr corticothérapie (prednisolone) per os à $1 \mathrm{mg} / \mathrm{kg}$ avec deux aérosols de budésonide par jour et un contrôle ORL le lendemain. La patiente est réadmise le surlendemain en raison de la dysphagie persistante et surveillée en unité d'hospitalisation de courte durée pendant 24 heures. Les douleurs s'amendent progressivement. La fracture de la styloïde radiale nécessitant une prise en charge chirurgicale, celle-ci est faite sous anesthésie locorégionale par bloc axillaire afin de ne pas majorer les douleurs cervicales du fait de l'intubation lors de l'anesthésie générale.

\section{Discussion}

Anatomiquement, l'os hyoïde est localisé en regard de la vertèbre $\mathrm{C} 3$, entre le cartilage thyroïdien et la mandibule. Il est suspendu par les ligaments stylohyoïdiens ainsi que les muscles s'attachant à la mandibule, aux processus styloïdiens, au cartilage thyroïdien et au manubrium et scapulas. Il est composé de cinq parties, le corps, deux grandes et deux petites cornes. Lors de l'hyperextension, l'os est alors exposé. La fracture de l'os hyoïde est bien connue dans le monde de la médecine légale, puisque le premier vecteur en est la strangulation ou la pendaison [4]. Les mécanismes secondaires responsables sont bien plus rares et représentés par les traumatismes directs ou indirects lors d'accidents de la voie publique associés ou non à des traumatismes faciaux multiples [5]. Certains sports comme le hockey, le taekwondo ou le football peuvent également être mis en cause en raison de l'exposition de l'os hyoïde aux impacts directs $[3-4,6]$. Le diagnostic en est difficile, en raison de la faible incidence et de la multitude de présentations possibles allant de la douleur antérieure cervicale à la dyspnée [1-2]. Le diagnostic se fait généralement sur une association d'éléments cliniques, imagerie et laryngoscopie [4]. Les fractures par hyperextension sont reconnues depuis le milieu du $\mathrm{XIX}^{\mathrm{e}}$ siècle, soit chez des sujets âgés souffrant d'ostéoporose, soit chez des sujets plus jeunes pour lesquels l'ossification entre le corps et la grande corne n'a pas encore été effectuée, ce qui a priori n'est pas le cas chez cette patiente de 45 ans 


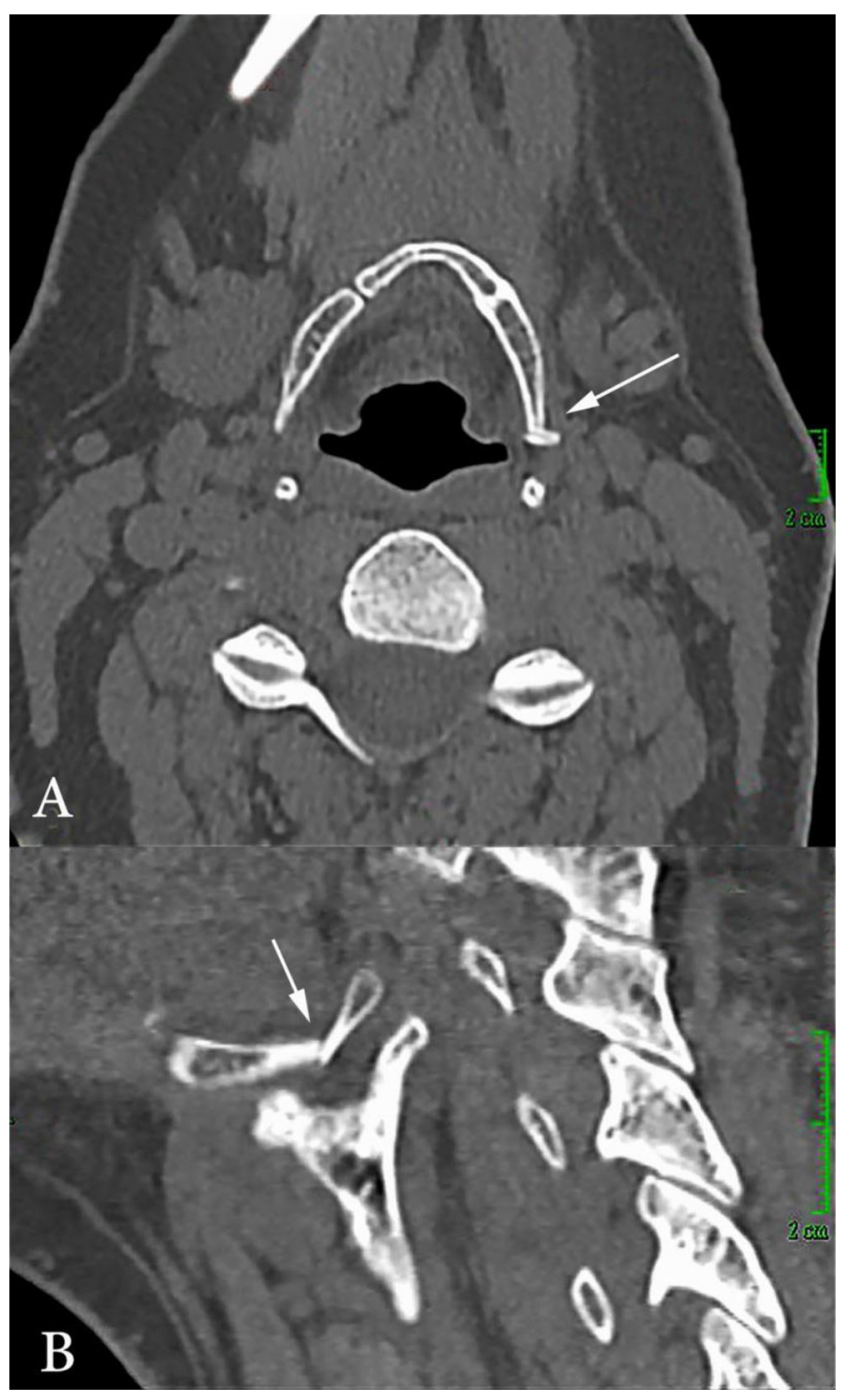

Fig. 1 Tomodensitométrie mettant en évidence la fracture de la corne gauche de l'os hyoïde (désignée par les flèches, que l'on retrouve en coupe axiale [A] et en coupe sagittale [B]) avec angulation vers le haut sans désinsertion (il s'agit d'une " fracture plicature », avec respect de continuité). L'angulation est d'environ $120^{\circ}$ à gauche (plus fermé) contre $140^{\circ}$ à droite entre le corps et les cornes principales postérolatérales de l'os hyoïde. Il n'y a pas de décalage dans le plan axial, mais un léger déplacement médial de la corne gauche

sans antécédent d'ostéoporose [3,7]. D'autres cas sporadiques de ce type de fracture ont été identifiés lors de chutes à vélo. Dans ces cas, la lanière du casque était impliquée ; or, notre patiente était non casquée $[2,8]$. Des cas de fracture de l'os hyoïde ont également été décrits lors d'accidents sur la voie publique chez des motards, mais toujours accompagnés de fractures maxillofaciales plus complexes, associant souvent des fractures de Lefort, ce qui ne correspond pas non plus à la cinétique ni à la gravité des lésions de notre patiente [9]. Concernant la thérapeutique, étant donné la bonne tolérance clinique de la patiente, les corticoïdes ont été choisis à visée anti-inflammatoire et antalgique, accompagnés d'une surveillance hospitalière. Une revue reprenant 36 articles déjà publiés a étudié la prise en charge de ces fractures, et conclut que le traitement conservateur semble être le plus fréquent, ce qui correspond au traitement de notre patiente [1]. Cependant, au vu de la faible fréquence de ce type de lésions et donc du nombre limité d'articles à ce sujet, la rédaction de recommandations solides reste difficile.

En conclusion, nous n'avons pas trouvé dans la littérature d'autre description de fracture de cet os par hyperextension du cou, sans impact direct ni fracture faciale associée, chez un adulte non ostéoporotique. Les fractures de cet os étant rares et méconnues donc possiblement sous-diagnostiquées — d'autant plus lors d'un mécanisme par hyperextension il est nécessaire d'être attentif lors de traumatismes cervicaux d'apparence bénigne.

Conflit d'intérêt : les auteurs déclarent ne pas avoir de lien d'intérêt.

\section{Références}

1. Ramchand T, Choudhry O, Shukla P, et al (2012) Management of hyoid bone fractures. Otolaryngol Head Neck Surg 147:204-08

2. Weingart S, Trueger S, Wong N, et al (2016) In reply: the risk of a bicycle helmet: hyoid bone fracture. Ann Emerg Med 67:144-45

3. Chowdhury R, Crocco A, El-Hakim H (2005) An isolated hyoid fracture secondary to sport injury. Int J Pediatr Otorhinolaryngol 69:411-14

4. Porr J, Laframboise M, Kazemi M (2012) Traumatic hyoid bone fracture - a case report and review of the literature. J Can Chiropr Assoc 56:269-74

5. Dalati T (2005) Isolated hyoid bone fracture. Int J Oral Maxillofac Surg 34:449-52

6. Cutuk A, Bissell B, Schmidt P, et al (2011) Isolated hyoid bone fractures in collegiate football players: a case series and review of the literature. Sports Health 4:51-6

7. Padgham N (1988) Hyperextension fracture of the hyoid bone. J Laryngol Otol 102:1062-63

8. Kuo L, Lin H, Chen C, et al (2008) Traumatic hyoid bone fracture in patient wearing a helmet: a case report. Am J Emerg Med 26:251.e1-e2

9. Keerthi R, Quadri A (2015) Hyoid bone fracture: associated with head and neck arauma-a rare case report. J Maxillofac Oral Surg 15:249-52 